\title{
En este número
}

\author{
Enrique Murcio-Pérez* \\ Departamento de Endoscopia Gastrointestinal, Centro Médico Nacional Siglo XXI, Instituto Mexicano del Seguro Social, Ciudad de México, México
}

"La inteligencia consiste no solo en el conocimiento, sino también en la destreza de aplicar los conocimientos en la práctica."

Aristóteles

El nuevo número de Endoscopia cumple con el objetivo de compartir el conocimiento y experiencia generada en Latinoamérica con procedimientos endoscópicos de diferente naturaleza y en diferentes tipos de pacientes. Nuestro medio socioeconómico hace que en ocasiones las patologías que nos afecten sean diferentes a las del resto del mundo y es importante tener en la mente dicho escenario. Un ejemplo claro es el caso de ascariasis hepatobiliar. ¿Es necesaria la endoscopia para su tratamiento o la terapia antihelmíntica es suficiente? Paredes-Soto, et al. nos comparten la información acerca de su diagnóstico y tratamiento en la sección de caso clínico.

Otra muestra de la importancia de evaluar resultados en otro tipo de población es el estudio de Cadena-León, et al., quienes presentan su experiencia con el uso de polvo hemostático en población pediátrica. A pesar del escaso número de pacientes incluidos, sus resultados evidencian la efectividad y seguridad de este tipo de terapia.

Por otro lado, un centro de referencia comparte su experiencia con el uso de balón intragástrico ajustable para la reducción de peso. Sin duda la obesidad es un problema de salud pública en México y el acceso a cirugía bariátrica para estos pacientes es muy bajo, lo que justifica determinar la utilidad de dichos dispositivos. Cano-Zepeda, et al. Utilizaron un balón intragástrico que puede permanecer en el estómago por 12 meses, esto se asoció a una disminución significativa del exceso de peso. Es relevante mencionar que no ocurrieron complicaciones mayores. Sin duda que la preocupación por la reganancia de peso tras la retirada del dispositivo y el manejo posterior de estos pacientes sigue siendo motivo de debate respecto a la utilidad o futilidad del procedimiento y ello es discutido por los autores.

Les invitamos a revisar el resto de los estudios incluidos en este número de la revista de Endoscopia esperando sea satisfactoria su lectura.
Disponible en internet: 06-09-2019

Endoscopia. 2019;31:99

www.endoscopia-ameg.com

0188-9893/@ 2019. Asociación Mexicana de Endoscopia Gastrointestinal, publicado por Permanyer México SA de CV, todos los derechos reservados. 